\section{MS4-P11 Evaluation of MRSAD phasing protocols}

Riccardo Pederzoli ${ }^{1}$, Fabio Dall'Antonia ${ }^{1}$, Isabel Bento ${ }^{1}$, Michele Cianci $^{1}$, Thomas R. Schneider ${ }^{1}$

1. EMBL (European Molecular Biology Laboratory) - c/o DESY, Building 25a, Notkestrasse 85, 22607, Hamburg, Germany

email: rpederzoli@embl-hamburg.de

Molecular Replacement in combination with Single Anomalous Diffraction (MRSAD) is a crystallographic phasing method that can lead to structure solution starting from weak anomalous signal and/or poor MR search models, where both the SAD and MR methods alone would fail. The advent of MRSAD has been triggered by the need to reduce the MR intrinsic model bias, in particular at low resolution, and by the increasing availability of high-resolution structures for components of larger biological complexes.

To explore the capabilities and limitations of currently available MRSAD protocols, we have tested these on known crystal structures where we assume that only a part of the crystallographic unit cell is known and can be placed by molecular replacement.

Our model system is $\mathrm{Cdc} 23^{\mathrm{NTerm}}$, a dimeric protein of which the structure has been recently determined via S-SAD at $3.1 \AA$ resolution ${ }^{1}$. Also, a monomeric, $1.9 \AA$-resolution structure has been obtained in 2013 by Se-SAD ${ }^{2}$. MRSAD has been tested on the low-resolution structure using different search models. The pipeline involves MR- and MRSAD-phasing, followed by model building/density modification with three different programs. To assess the results, the deposited PDB model has been used as a reference. The success of the protocol has been judged on the number of residues built into the electron density map, on the phase quality as evaluated via the Mean Phase Error (MPE) and real-space map correlation coefficients against the reference.

Numerically, the improvements from MR to MRSAD are limited to a few degrees in terms of MPE. However, the visual inspection of electron density maps and the analysis of the real space correlation coefficients have shown that the electron densities produced with MRSAD phases are clearly superior to those produced from MR or SAD phases alone.

As a next step of the study, we will apply the same strategy to larger macromolecular complexes.

1. Cianci, M. et al. (2016), Acta Cryst. D72, 403-412

2. Zhang, Z. et al. (2013), J. Mol. Biol. 425, 4236-4248.

\section{MS4-P12 Advances in nucleic acid model} building with ARP/wARP 7.6

Grzegorz Chojnowski ${ }^{1}$, Victor Lamzin ${ }^{1}$

1. European Molecular Biology Laboratory (EMBL), c/o DESY, Notkestrasse 85, Hamburg 22607, Germany

email: gchojnowski@embl-hamburg.de

Nucleic acids perform a variety of functions in a living cell beyond their protein coding capacity. In particular, non-coding RNAs (ncRNAs) have been identified to be involved in many cellular processes ranging from the regulation of gene transcription to the catalysis of chemical reactions. Many known ncRNAs form compact three-dimensional structures, therefore understanding of their function in the cell requires knowledge of their molecular structure.

Over the years the ARP/wARP software has been continuously advancing and used by more than 15,000 researchers for building structural models of proteins with or without nucleic acids or small-molecule ligands. Nucleic acid model building was introduced in ARP/wARP relatively recently and it is algorithmically analogous to the protein chain tracing. In brief, the procedure starts with the detection of candidate positions of backbone phosphates. Subsequently, phosphate-ribose-phosphate units are placed in electron density with the use of graph theory and pattern recognition approaches. Finally, the bases are added to the backbone and the built chain fragments undergo restrained real-space refinement in the electron density.

Now we present a considerable revision of the nucleic acid model building module in ARP/wARP. The phosphate group detection, which is crucial for the successful polynucleotide tracing, has been complemented with the new machine learning algorithm. It now performs better at lower resolution and in poorly defined density regions. Moreover, the tracing of the nucleic acid backbone has been advanced in an iterative manner, similar to the protein chain tracing. Compared to previous version of ARP/wARP (7.5) the new revision resulted in an overall increase in the number of nucleotides built by $15 \%$, while maintaining the high level of accuracy of the built models.

Keywords: nucleic acids, model building

Keywords: Molecular Replacement, Single Anomalous Diffraction, MRSAD 